\title{
APPLICATIONS OF THE POINCARÉ INEQUALITY TO EXTENDED KANTOROVICH METHOD
}

DER-CHEN CHANG, TRISTAN NGUYEN, GANG WANG, AND NORMAN M. WERELEY

Received 3 February 2005; Revised 2 March 2005; Accepted 18 April 2005

We apply the Poincare inequality to study the extended Kantorovich method that was used to construct a closed-form solution for two coupled partial differential equations with mixed boundary conditions.

Copyright (c) 2006 Der-Chen Chang et al. This is an open access article distributed under the Creative Commons Attribution License, which permits unrestricted use, distribution, and reproduction in any medium, provided the original work is properly cited.

\section{Introduction}

Let $\Omega \subset \mathbb{R}^{n}$ be a Lipschitz domain in $\mathbb{R}^{n}$. Consider the Dirichlet space $H_{0}^{1}(\Omega)$ which is the collection of all functions in the Sobolev space $L_{1}^{2}(\Omega)$ such that

$$
H_{0}^{1}(\Omega)=\left\{u \in L^{2}(\Omega):\left.u\right|_{\partial \Omega}=0,\|u\|_{L^{2}}+\sum_{k=1}^{n}\left\|\frac{\partial u}{\partial x_{k}}\right\|_{L^{2}}<\infty\right\} .
$$

The famous Poincaré inequality can be stated as follows: for $u \in H_{0}^{1}(\Omega)$, then there exists a universal constant $C$ such that

$$
\int_{\Omega} u^{2}(\mathbf{x}) d \mathbf{x} \leq C \sum_{k=1}^{n} \int_{\Omega}\left|\frac{\partial u}{\partial x_{k}}\right|^{2} d \mathbf{x}
$$

One of the applications of this inequality is to solve the modified version of the Dirichlet problem (see, John [5, page 97]): find a $v \in H_{0}^{1}(\Omega)$ such that

$$
(u, v)=\int_{\Omega}\left[\sum_{k=1}^{n} \frac{\partial u}{\partial x_{k}} \frac{\partial v}{\partial x_{k}}\right] d \mathbf{x}=\int_{\Omega} u(\mathbf{x}) f(\mathbf{x}) d \mathbf{x},
$$


2 Poincaré inequality and Kantorovich method

where $\mathbf{x}=\left(x_{1}, \ldots, x_{n}\right)$ with a fixed $f \in C(\bar{\Omega})$. Then the function $v$ in (1.3) satisfied the boundary value problem

$$
\begin{gathered}
\Delta v=-f, \quad \text { in } \Omega \\
v=0, \quad \text { on } \partial \Omega .
\end{gathered}
$$

In this paper, we will use the Poincare inequality to study the extended Kantorovich method, see [6]. This method has been used extensively in many engineering problems, for example, readers can consult papers $[4,7,8,11,12]$, and the references therein. Let us start with a model problem, see [8]. For a clamped rectangular box $\Omega=\prod_{k=1}^{n}\left[-a_{k}, a_{k}\right]$, subjected to a lateral distributed load, $\mathscr{P}(\mathbf{x})=\mathscr{P}\left(x_{1}, \ldots, x_{n}\right)$, the principle of virtual displacements yields

$$
\prod_{\ell=1}^{n} \int_{-a_{\ell}}^{a_{\ell}}\left[\eta \nabla^{4} \Phi-\mathscr{P}\right] \delta \Phi D \mathbf{x}=0
$$

where $\Phi$ is the lateral deflection which satisfies the boundary conditions, $\eta$ is the flexural rigidity of the box, and

$$
\nabla^{4}=\sum_{k=1}^{n} \frac{\partial^{4}}{\partial x_{k}^{4}}+\sum_{j \neq k} 2 \frac{\partial^{4}}{\partial x_{j}^{2} \partial x_{k}^{2}}
$$

Since the domain $\Omega$ is a rectangular box, it is natural to assume the deflection in the form

$$
\Phi(\mathbf{x})=\Phi_{k_{1} \cdots k_{n}}(\mathbf{x})=\prod_{\ell=1}^{n} f_{k_{\ell}}\left(x_{\ell}\right),
$$

it follows that when $f_{k_{2}}\left(x_{2}\right) \cdots f_{k_{n}}\left(x_{n}\right)$ is prescribed a priori, (1.5) can be rewritten as

$$
\int_{-a_{1}}^{a_{1}}\left[\prod_{\ell=2}^{n} \int_{-a_{\ell}}^{a_{\ell}}\left(\eta \nabla^{4} \Phi_{k_{1}} \cdots k_{n}-\mathscr{P}\right) f_{k_{\ell}}\left(x_{\ell}\right) d x_{\ell}\right] \delta f_{k_{1}}\left(x_{1}\right) d x_{1}=0 .
$$

Equation (1.8) is satisfied when

$$
\prod_{\ell=2}^{n} \int_{-a_{\ell}}^{a_{\ell}}\left(\eta \nabla^{4} \Phi_{k_{1} \cdots k_{n}}-\mathscr{P}\right) f_{k_{\ell}}\left(x_{\ell}\right) d x_{\ell}=0
$$

Similarly, when $\prod_{\ell=1, \ell \neq m}^{n} f_{k_{\ell}}\left(x_{\ell}\right)$ is prescribed a priori, (1.5) can be rewritten as

$$
\int_{-a_{m}}^{a_{m}}\left[\prod_{\ell=1, \ell \neq m}^{n} \int_{-a_{\ell}}^{a_{\ell}}\left(\eta \nabla^{4} \Phi_{k_{1} \cdots k_{n}}-\mathscr{P}\right) f_{k_{\ell}}\left(x_{\ell}\right) d x_{\ell}\right] \delta f_{k_{m}}\left(x_{m}\right) d x_{m}=0 .
$$

It is satisfied when

$$
\prod_{\ell=1, \ell \neq m}^{n} \int_{-a_{\ell}}^{a_{\ell}}\left(\eta \nabla^{4} \Phi_{k_{1} \cdots k_{n}}-\mathscr{P}\right) f_{k_{\ell}}\left(x_{\ell}\right) d x_{\ell}=0 .
$$


It is known that (1.9) and (1.11) are called the Galerkin equations of the extended Kantorovich method. Now we may first choose

$$
f_{20}\left(x_{2}\right) \cdots f_{n 0}\left(x_{n}\right)=\prod_{\ell=2}^{n} c_{\ell}\left(\frac{x_{\ell}^{2}}{a_{\ell}^{2}}-1\right)^{2} .
$$

Then $\Phi_{10 \cdots 0}(\mathbf{x})=f_{11}\left(x_{1}\right) f_{20}\left(x_{2}\right) \cdots f_{n 0}\left(x_{n}\right)$ satisfies the boundary conditions

$$
\Phi_{10 \cdots 0}=0, \quad \frac{\partial \Phi_{10 \cdots 0}}{\partial x_{\ell}}=0 \quad \text { at } x_{\ell}= \pm a_{\ell}, x_{1} \in\left[-a_{1}, a_{1}\right]
$$

for $\ell=2, \ldots, n$. Now (1.9) becomes

$$
\prod_{\ell=2}^{n} c_{\ell} \int_{-a_{\ell}}^{a_{\ell}}\left(\nabla^{4} \Phi_{10 \cdots 0}-\frac{\mathscr{P}}{\eta}\right)\left(\frac{x_{\ell}^{2}}{a_{\ell}^{2}}-1\right)^{2} d x_{\ell}=0,
$$

which yields

$$
C_{4} \frac{d^{4} f_{11}}{d x^{4}}+C_{2} \frac{d^{2} f_{11}}{d x^{2}}+C_{0} f_{11}=B
$$

After solving the above ODE, we can use $f_{11}\left(x_{1}\right) \prod_{\ell=3}^{n} f_{\ell 0}\left(x_{\ell}\right)$ as a priori data and plug it into (1.10) to find $f_{21}\left(x_{2}\right)$. Then we obtain the function

$$
\Phi_{110 \cdots 0}(\mathbf{x})=f_{11}\left(x_{1}\right) f_{21}\left(x_{2}\right) f_{30}\left(x_{3}\right) \cdots f_{n 0}\left(x_{n}\right) .
$$

Continue this process until we obtain $\Phi_{1 \cdots 1}(\mathbf{x})=f_{11}\left(x_{1}\right) f_{21}\left(x_{2}\right) \cdots f_{n 1}\left(x_{n}\right)$ and therefore completes the first cycle. Next, we use $f_{21}\left(x_{2}\right) \cdots f_{n 1}\left(x_{n}\right)$ as our priori data and find $f_{12}\left(x_{1}\right)$. We continue this process and expect to find a sequence of "approximate solutions." The problem reduces to investigate the convergence of this sequence. Therefore, it is crucial to analyze (1.15). Moreover, from numerical point of view, we know that this sequence converges rapidly (see $[1,2]$ ). Hence, it is necessary to give a rigorous mathematical proof of this method.

\section{A convex linear functional on $H_{0}^{2}(\Omega)$}

Denote

$$
I[\phi]=\int_{\Omega}\left\{|\Delta \phi|^{2}-2 \mathscr{P}(\mathbf{x}) \phi(\mathbf{x})\right\} d \mathbf{x}
$$

for $\Omega \subset \mathbb{R}^{n}$ a bounded Lipschitz domain. Here $\mathbf{x}=\left(x_{1}, \ldots, x_{n}\right)$. As usual, denote

$$
D^{2} \phi=\left[\begin{array}{cc}
\frac{\partial^{2} \phi}{\partial x^{2}} & \frac{\partial^{2} \phi}{\partial x \partial y} \\
\frac{\partial^{2} \phi}{\partial y \partial x} & \frac{\partial^{2} \phi}{\partial y^{2}}
\end{array}\right] .
$$


4 Poincaré inequality and Kantorovich method

For $\Omega \subset \mathbb{R}^{2}$, we define the Lagrangian function $L$ associated to $I[\phi]$ as follows:

$$
\begin{gathered}
L: \Omega \times \mathbb{R} \times \mathbb{R}^{2} \times \mathbb{R}^{4} \longrightarrow \mathbb{R}, \\
(x, y ; z ; X, Y ; U, V, S, W) \longmapsto(U+V)^{2}-2 \mathscr{P}(x, y) z,
\end{gathered}
$$

where $\mathscr{P}(x, y)$ is a fixed function on $\Omega$ which shows up in the integrand of $I[\phi]$. With the above definitions, we have

$$
L\left(x, y ; \phi ; \nabla \phi ; D^{2} \phi\right)=|\Delta \phi|^{2}-2 \mathscr{P}(x, y) \phi(x, y),
$$

where we have identified

$$
\begin{gathered}
z \longleftrightarrow \phi(x, y), \quad X \longleftrightarrow \frac{\partial \phi}{\partial x}, \quad Y \longleftrightarrow \frac{\partial \phi}{\partial y}, \\
U \longleftrightarrow \frac{\partial^{2} \phi}{\partial x^{2}}, \quad V \longleftrightarrow \frac{\partial^{2} \phi}{\partial y^{2}}, \quad S \longleftrightarrow \frac{\partial^{2} \phi}{\partial y \partial x}, \quad W \longleftrightarrow \frac{\partial^{2} \phi}{\partial x \partial y} .
\end{gathered}
$$

We also set $H_{0}^{2}(\Omega)$ to be the class of all square integrable functions such that

$$
H_{0}^{2}(\Omega)=\left\{\psi \in L^{2}(\Omega): \sum_{|\mathbf{k}| \leq 2}\left\|\frac{\partial^{\mathbf{k}} \psi}{\partial \mathbf{x}^{\mathbf{k}}}\right\|_{L^{2}}<\infty,\left.\psi\right|_{\partial \Omega}=0,\left.\nabla \psi\right|_{\partial \Omega}=\mathbf{0}\right\} .
$$

Fix $(x, y) \in \Omega$. We know that

$$
\nabla L(x, y ; z ; X, Y ; U, V, S, W)=\left[\begin{array}{lllllll}
-2 \mathscr{P}(x, y) & 0 & 0 & 2(U+V) & 2(U+V) & 0 & 0
\end{array}\right]^{T} .
$$

Because the convexity of the function $L$ in the remaining variables, then for all $(\tilde{z} ; \tilde{X}, \tilde{Y} ; \tilde{U}$, $\tilde{V}, \widetilde{S}, \widetilde{W}) \in \mathbb{R} \times \mathbb{R}^{2} \times \mathbb{R}^{4}$, one has

$$
\begin{aligned}
L(x, y ; \tilde{z} ; \tilde{X}, \tilde{Y} ; \tilde{U}, \tilde{V}, \tilde{S}, \widetilde{W}) \geq & L(x, y ; z ; X, Y ; U, V, S, W)-2 \mathscr{P}(x, y)(\tilde{z}-z) \\
& +2(U+V)[(\tilde{U}-U)+(\tilde{V}-V)] .
\end{aligned}
$$

In particular, one has, with $\tilde{z}=\tilde{\phi}(x, y)$,

$$
\begin{aligned}
L\left(x, y ; \tilde{\phi} ; \nabla \tilde{\phi} ; D^{2} \tilde{\phi}\right) \geq & L\left(x, y ; \phi ; \nabla \phi ; D^{2} \phi\right)+2 \Delta \phi[\nabla \tilde{\phi}-\nabla \phi] \\
& -2 \mathscr{P}(x, y)(\tilde{\phi}-\phi) .
\end{aligned}
$$

This implies that

$$
|\Delta \tilde{\phi}|^{2}-2 \mathscr{P}(x, y) \tilde{\phi} \geq|\Delta \phi|^{2}-2 \mathscr{P}(x, y) \phi+2 \Delta \phi[\Delta \tilde{\phi}-\Delta \phi]-2 \mathscr{P}(x, y)[\tilde{\phi}-\phi]
$$

If instead we fix $(x, y ; z) \in \Omega \times \mathbb{R}$, then

$$
\begin{aligned}
L(x, y ; \tilde{z} ; \tilde{X}, \tilde{Y} ; \tilde{U}, \tilde{V}, \tilde{S}, \widetilde{W}) \geq & L(x, y ; \tilde{z} ; X, Y ; U, V, S, W) \\
& +2(U+V)[(\tilde{U}-U)+(\tilde{V}-V)] .
\end{aligned}
$$


This implies that

$$
L\left(x, y ; \tilde{\phi} ; \nabla \tilde{\phi} ; D^{2} \tilde{\phi}\right) \geq L\left(x, y ; \tilde{\phi} ; \nabla \phi ; D^{2} \phi\right)+2 \Delta \phi[\nabla \tilde{\phi}-\nabla \phi]
$$

Therefore,

$$
|\Delta \tilde{\phi}|^{2}-2 \mathscr{P}(x, y) \tilde{\phi} \geq|\Delta \phi|^{2}-2 \mathscr{P}(x, y) \tilde{\phi}+2 \Delta \phi[\Delta \tilde{\phi}-\Delta \phi]
$$

Lemma 2.1. Suppose either

(1) $\phi \in H_{0}^{2}(\Omega) \cap C^{4}(\Omega)$ and $\eta \in C_{c}^{1}(\Omega)$; or

(2) $\phi \in H_{0}^{2}(\Omega) \cap C^{3}(\bar{\Omega}) \cap C^{4}(\Omega)$ and $\eta \in H_{0}^{2}(\Omega)$.

Let $\delta I[\phi ; \eta]$ denote the first variation of I at $\phi$ in the direction $\eta$, that is,

$$
\delta I[\phi ; \eta]=\lim _{\varepsilon \rightarrow 0} \frac{I[\phi+\varepsilon \eta]-I[\phi]}{\varepsilon} .
$$

Then

$$
\delta I[\phi ; \eta]=2 \int_{\Omega}\left(\Delta^{2} \phi-\mathscr{P}(x, y)\right) \eta d x d y .
$$

Proof. We know that

$$
I[\phi+\varepsilon \eta]-I[\phi]=2 \varepsilon \int_{\Omega}[\Delta \phi \Delta \eta-\mathscr{P} \eta] d x d y+\varepsilon^{2} \int_{\Omega}(\Delta \eta)^{2} d x d y .
$$

Hence,

$$
\varepsilon I[\phi ; \eta]=2 \int_{\Omega}[\Delta \phi \Delta \eta-\mathscr{P} \eta] d x d y
$$

If either assumption (1) or (2) holds, we can apply Green's formula to a Lipschitz domain $\Omega$ to obtain

$$
\int_{\Omega}(\Delta \phi \Delta \eta) d x d y=\int_{\Omega} \eta\left(\Delta^{2} \phi\right) d x d y+\int_{\partial \Omega}\left[\frac{\partial \eta}{\partial \vec{n}} \Delta \phi-\eta \frac{\partial}{\partial \vec{n}} \Delta \phi\right] d x d y,
$$

where $\partial / \partial \vec{n}$ is the derivative in the direction normal to $\partial \Omega$. Since either $\eta \in C_{c}^{1}(\Omega)$ or $\eta \in H_{0}^{2}(\Omega)$, the boundary term vanishes, which proves the lemma.

Lemma 2.2. Let $\phi \in H_{0}^{2}(\Omega)$. Then

$$
\|\phi\|_{H_{0}^{2}(\Omega)} \approx\|\Delta \phi\|_{L^{2}(\Omega)}
$$

Proof. The function $\phi \in H_{0}^{2}(\Omega)$ implies that there exists a sequence $\left\{\phi_{k}\right\} \subset C_{c}^{\infty}(\Omega)$ such that $\lim _{k \rightarrow \infty} \phi_{k}=\phi$ in $H_{0}^{2}$-norm. From a well-known result for the Calderón-Zygmund operator (see, Stein [10, page 77]), one has

$$
\left\|\frac{\partial^{2} f}{\partial x_{j} \partial x_{\ell}}\right\|_{L^{p}} \leq C\|\Delta f\|_{L^{p}}, \quad j, \ell=1, \ldots, n
$$


6 Poincaré inequality and Kantorovich method

for all $f \in C_{c}^{2}\left(\mathbb{R}^{n}\right)$ and $1<p<\infty$. Here $C$ is a constant that depends on $n$ only. Applying this result to each $\phi_{k}$, we obtain

$$
\left\|\frac{\partial^{2} \phi_{k}}{\partial x^{2}}\right\|_{L^{2}(\Omega)},\left\|\frac{\partial^{2} \phi_{k}}{\partial x \partial y}\right\|_{L^{2}(\Omega)},\left\|\frac{\partial^{2} \phi_{k}}{\partial y^{2}}\right\|_{L^{2}(\Omega)} \leq C\left\|\Delta \phi_{k}\right\|_{L^{2}(\Omega)} .
$$

Taking the limit, we conclude that

$$
\left\|\frac{\partial^{2} \phi}{\partial x^{2}}\right\|_{L^{2}(\Omega)},\left\|\frac{\partial^{2} \phi}{\partial x \partial y}\right\|_{L^{2}(\Omega)},\left\|\frac{\partial^{2} \phi}{\partial y^{2}}\right\|_{L^{2}(\Omega)} \leq C\|\Delta \phi\|_{L^{2}(\Omega)} .
$$

Applying Poincaré inequality twice to the function $\phi \in H_{0}^{2}(\Omega)$, we have

$$
\begin{aligned}
\|\phi\|_{L^{2}(\Omega)} & \leq C_{1}\|\nabla \phi\|_{L^{2}(\Omega)} \\
& \leq C_{2}\left(\left\|\frac{\partial^{2} \phi}{\partial x^{2}}\right\|_{L^{2}(\Omega)}+\left\|\frac{\partial^{2} \phi}{\partial x \partial y}\right\|_{L^{2}(\Omega)}+\left\|\frac{\partial^{2} \phi}{\partial y^{2}}\right\|_{L^{2}(\Omega)}\right) \\
& \leq C\|\Delta \phi\|_{L^{2}(\Omega) .}
\end{aligned}
$$

Hence, $\|\phi\|_{L^{2}(\Omega)} \leq C\|\Delta \phi\|_{L^{2}(\Omega)}$. The reverse inequality is trivial. The proof of this lemma is therefore complete.

Lemma 2.3. Let $\left\{\phi_{k}\right\}$ be a bounded sequence in $H_{0}^{2}(\Omega)$. Then there exist $\phi \in H_{0}^{2}(\Omega)$ and a subsequence $\left\{\phi_{k_{j}}\right\}$ such that

$$
I[\phi] \leq \liminf I\left[\phi_{k_{j}}\right] .
$$

Proof. By a weak compactness theorem for reflexive Banach spaces, and hence for Hilbert spaces, there exist a subsequence $\left\{\phi_{k_{j}}\right\}$ of $\left\{\phi_{k}\right\}$ and $\phi$ in $H_{0}^{2}(\Omega)$ such that $\phi_{k_{j}} \rightarrow \phi$ weakly in $H_{0}^{2}(\Omega)$. Since

$$
H_{0}^{2}(\Omega) \subset H_{0}^{1}(\Omega) \subset \subset L^{2}(\Omega),
$$

by the Sobolev embedding theorem, we have

$$
\phi_{k_{j}} \rightarrow \phi \quad \text { in } L^{2}(\Omega)
$$

after passing to yet another subsequence if necessary.

Now fix $\left(x, y, \phi_{k_{j}}(x, y)\right) \in \mathbb{R}^{2} \times \mathbb{R}$ and apply inequality (2.13), we have

$$
\left|\Delta \phi_{k_{j}}\right|^{2}-2 \mathscr{P}(x, y) \phi_{k_{j}}(x, y) \geq|\Delta \phi|^{2}-2 \mathscr{P}(x, y) \phi_{k_{j}}(x, y)+2 \Delta \phi\left[\Delta \phi_{k_{j}}-\Delta \phi\right] .
$$

This implies that

$$
I\left[\phi_{k_{j}}\right] \geq \int_{\Omega}\left[|\Delta \phi|^{2}-2 \mathscr{P}(x, y) \phi_{k_{j}}\right] d x d y+2 \int_{\Omega} \Delta \phi \cdot\left[\Delta \phi_{k_{j}}-\Delta \phi\right] d x d y .
$$

But $\phi_{k_{j}} \rightarrow \phi$ in $L^{2}(\Omega)$, hence

$$
\int_{\Omega}\left[|\Delta \phi|^{2}-2 \mathscr{P}(x, y) \phi_{k_{j}}\right] d x d y \longrightarrow \int_{\Omega}\left[|\Delta \phi|^{2}-2 \mathscr{P}(x, y) \phi\right] d x d y=I[\phi] .
$$


Besides $\phi_{k_{j}} \rightarrow \phi$ weakly in $H_{0}^{2}(\Omega)$ implies that

$$
\int_{\Omega} \Delta \phi \cdot\left[\Delta \phi_{k_{j}}-\Delta \phi\right] d x d y \longrightarrow 0 .
$$

It follows that when taking limit

$$
I[\phi] \leq \liminf _{j} I\left[\phi_{k_{j}}\right] .
$$

This completes the proof of the lemma.

Remark 2.4. The above proof uses the convexity of $L(x, y ; z ; X, Y ; U, V, S, W)$ when $(x, y$; $z)$ is fixed. We already remarked at the beginning of this section that when $(x, y)$ is fixed, $L(x, y ; z ; X, Y ; U, V, S, W)$ is convex in the remaining variables, including the $z$-variable. That is, we are not required to utilize the full strength of the convexity of $L$ here.

\section{The extended Kantorovich method}

Now, we shift our focus to the extended Kantorovich method for finding an approximate solution to the minimization problem

$$
\min _{\phi \in H_{0}^{2}(\Omega)} I[\phi]
$$

when $\Omega=[-a, a] \times[-b, b]$ is a rectangular region in $\mathbb{R}^{2}$. In the sequel, we will write $\phi(x, y)$ (resp., $\phi_{k}(x, y)$ ) as $f(x) g(y)$ (resp., $\left.f_{k}(x) g_{k}(y)\right)$ interchangeably as notated in Kerr and Alexander [8]. More specifically, we will study the extended Kantorovich method for the case $n=2$, which has been used extensively in the analysis of stress on rectangular plates. Equivalently, we will seek for an approximate solution of the above minimization problem in the form $\phi(x, y)=f(x) g(y)$ where $f \in H_{0}^{2}([-a, a])$ and $g \in H_{0}^{2}([-b, b])$.

To phrase this differently, we will search for an approximate solution in the tensor product Hilbert spaces $H_{0}^{2}([-a, a]) \hat{\otimes} H_{0}^{2}([-b, b])$, and all sequences $\left\{\phi_{k}\right\},\left\{\phi_{k_{j}}\right\}$ involved hereinafter reside in this Hilbert space. Without loss of generality, we may assume that $\Omega=[-1,1] \times[-1,1]$ for all subsequent results remain valid for the general case where $\Omega=[-a, a] \times[-b, b]$ by approximate scalings/normalizing of the $x$ and $y$ variables. As in [8], we will treat the special case $\mathscr{P}(x, y)=\gamma$, that is, we assume that the load $\mathscr{P}(x, y)$ is distributed equally on a given rectangular plate.

To start the extended Kantorovich scheme, we first choose $g_{0}(y) \in H_{0}^{2}([-1,1]) \cap$ $C_{c}^{\infty}(-1,1)$, and find the minimizer $f_{1}(x) \in H_{0}^{2}([-1,1])$ of the functional:

$$
\begin{aligned}
I\left[f g_{0}\right]= & \int_{\Omega}\left[\left|\Delta\left(f g_{0}\right)\right|^{2}-2 \gamma f(x) g_{0}(y)\right] d x d y \\
= & \int_{\Omega}\left[g_{0}^{2}\left(f^{\prime \prime}\right)^{2}+2 f f^{\prime \prime} g_{0} g_{0}^{\prime \prime}+f^{2}\left(g_{0}^{\prime \prime}\right)^{2}-2 \gamma f g_{0}\right] d x d y \\
= & \int_{-1}^{1}\left(f^{\prime \prime}\right)^{2} d x \int_{-1}^{1} g_{0}^{2} d y+2 \int_{-1}^{1}\left(g_{0}^{\prime}\right)^{2} d y \int_{-1}^{1}\left(f^{\prime}\right)^{2} d x \\
& +\int_{-1}^{1}\left(g_{0}^{\prime \prime}\right)^{2} d y \int_{-1}^{1} f^{2} d x-2 \gamma \int_{-1}^{1} g_{0} d y \int_{-1}^{1} f d x
\end{aligned}
$$


8 Poincaré inequality and Kantorovich method

where the last equality was obtained via the integration by parts of $f f^{\prime \prime}$ and $g_{0} g_{0}^{\prime \prime}$. Since $g_{0}$ has been chosen a priori; we can rewrite the functional $I$ as

$$
\begin{aligned}
J[f]= & \left\|g_{0}\right\|_{L^{2}}^{2} \int_{-1}^{1}\left(f^{\prime \prime}\right)^{2} d x+2\left\|g_{0}^{\prime}\right\|_{L^{2}}^{2} \int_{-1}^{1}\left(f^{\prime}\right)^{2} d x \\
& +\left\|g_{0}^{\prime \prime}\right\|_{L^{2}}^{2} \int_{-1}^{1} f^{2} d x-2 \gamma \int_{-1}^{1} g_{0}(y) d y \int_{-1}^{1} f d x
\end{aligned}
$$

for all $f \in H_{0}^{2}([-1,1])$. Now we may rewrite (3.3) in the following form:

$$
\begin{aligned}
J[f] & =\int_{-1}^{1}\left[C_{1}\left(f^{\prime \prime}\right)^{2}+C_{2}\left(f^{\prime}\right)^{2}+C_{3} f^{2}+C_{4} f\right] d x \\
& \equiv \int_{-1}^{1} K\left(x, f, f^{\prime}, f^{\prime \prime}\right) d x
\end{aligned}
$$

with $K: \mathbb{R} \times \mathbb{R} \times \mathbb{R} \times \mathbb{R} \rightarrow \mathbb{R}$ given by

$$
(x ; z ; V ; W) \longmapsto C_{1} W^{2}+C_{2} V^{2}+C_{3} z^{2}+C_{4} z,
$$

where

$$
C_{1}=\left\|g_{0}\right\|_{L^{2}}^{2}, \quad C_{2}=\left\|g_{0}^{\prime}\right\|_{L^{2}}^{2}, \quad C_{3}=\left\|g_{0}^{\prime \prime}\right\|_{L^{2}}^{2}, \quad C_{4}=-2 \gamma \int_{-1}^{1} g_{0}(y) d y .
$$

As long as $g_{0} \not \equiv 0$, as we have implicitly assumed, the Poincaré inequality implies that

$$
0<C_{1} \leq \alpha C_{2} \leq \beta C_{3}
$$

for some positive constants $\alpha$ and $\beta$, independent of $g_{0}$. Consequently, $K(x ; z ; V ; W)$ is a strictly convex function in variable $z, V, W$ when $x$ is fixed. In other words, $K$ satisfies

$$
\begin{aligned}
& K(x ; \tilde{z} ; \tilde{V} ; \widetilde{W})-K(x ; z ; V ; W) \\
& \quad \geq \frac{\partial K}{\partial z}(x ; z ; V ; W)(\tilde{z}-z)+\frac{\partial K}{\partial V}(x ; z ; V ; W)(\tilde{V}-V)+\frac{\partial K}{\partial W}(x ; z ; V ; W)(\widetilde{W}-W)
\end{aligned}
$$

for all $(x ; z ; V ; W)$ and $(x ; \tilde{z} ; \tilde{V} ; \widetilde{W})$ in $\mathbb{R}^{4}$, and the inequality becomes equality at $(x ; z ; V$; $W$ ) only if $\tilde{z}=z$, or $\tilde{V}=V$, or $\widetilde{W}=W$.

Proposition 3.1. Let $\mathscr{L}: \mathbb{R} \times \mathbb{R} \times \mathbb{R} \times \mathbb{R} \rightarrow \mathbb{R}$ be a $C^{\infty}$ function satisfying the following convexity condition:

$$
\begin{aligned}
& \mathscr{L}\left(x ; z+z^{\prime} ; V+V^{\prime} ; W+W^{\prime}\right)-\mathscr{L}(x ; z ; V ; W) \\
& \quad \geq \frac{\partial \mathscr{L}}{\partial z}(x ; z ; V ; W) z^{\prime}+\frac{\partial \mathscr{L}}{\partial V}(x ; z ; V ; W) V^{\prime}+\frac{\partial \mathscr{L}}{\partial W}(x ; z ; V ; W) W^{\prime}
\end{aligned}
$$


for all $(x ; z ; V ; W)$ and $\left(x ; z+z^{\prime} ; V+V^{\prime} ; W+W^{\prime}\right) \in \mathbb{R}^{4}$, with equality at $(x ; z ; V ; W)$ only if $z^{\prime}=0$, or $V^{\prime}=0$, or $W^{\prime}=0$. Also, let

$$
J[f]=\int_{\alpha}^{\beta} \mathscr{L}\left(x, f(x), f^{\prime}(x), f^{\prime \prime}(x)\right) d x, \quad \forall f \in H_{0}^{2}(\alpha, \beta) .
$$

Then

$$
J[f+\eta]-J[f] \geq \delta J[f, \eta], \quad \forall \eta \in C_{c}^{\infty}(\alpha, \beta)
$$

and equality holds only if $\eta \equiv 0$. Here $\delta J[f, \eta]$ is the first variation of $J$ at $f$ in the direction $\eta$. Proof. Condition (3.9) means that at each $x$,

$$
\begin{aligned}
& \mathscr{L}\left(x ; f+\eta ; f^{\prime}+\eta^{\prime} ; f^{\prime \prime}+\eta^{\prime \prime}\right)-\mathscr{L}\left(x ; f ; f^{\prime} ; f^{\prime \prime}\right) \\
& \quad \geq \frac{\partial \mathscr{L}}{\partial z}\left(x ; f ; f^{\prime} ; f^{\prime \prime}\right) \eta(x)+\frac{\partial \mathscr{L}}{\partial V}\left(x ; f ; f^{\prime} ; f^{\prime \prime}\right) \eta^{\prime}(x)+\frac{\partial \mathscr{L}}{\partial W}\left(x ; f ; f^{\prime} ; f^{\prime \prime}\right) \eta^{\prime \prime}(x)
\end{aligned}
$$

for all $\eta \in C_{c}^{\infty}(\alpha, \beta)$ with equality only if $\eta(x)=0$, or $\eta^{\prime}(x)=0$, or $\eta^{\prime \prime}(x)=0$. Equivalently, the equality holds in (3.12) at $x$ only if $\eta(x) \eta^{\prime}(x)=0$ or $\eta^{\prime \prime}(x)=0$. In other words,

$$
\eta^{\prime \prime}(x) \frac{d}{d x}\left(\eta^{2}(x)\right)=0 \text {. }
$$

Integrating (3.12) gives

$$
J[f+\eta]-J[f] \geq \int_{\alpha}^{\beta}\left[\frac{\partial \mathscr{L}}{\partial z} \eta+\frac{\partial \mathscr{L}}{\partial V} \eta^{\prime}+\frac{\partial \mathscr{L}}{\partial W} \eta^{\prime \prime}\right] d x=\delta J[f, \eta] .
$$

Now suppose there exists $\eta \in C_{c}^{\infty}(\alpha, \beta)$ such that (3.14) is an equality. Since $\mathscr{L}$ is a smooth function, this equality forces (3.12) to be a pointwise equality, which implies, in view of (3.13), that

$$
\eta^{\prime \prime}(x) \frac{d}{d x}\left(\eta^{2}(x)\right)=0, \quad \forall x
$$

If $\eta^{\prime \prime}(x) \equiv 0$, then $\eta^{\prime}(x)=$ constant which implies that $\eta^{\prime}(x) \equiv 0\left(\right.$ since $\left.\eta \in C_{c}^{\infty}(\alpha, \beta)\right)$. This tells us that $\eta \equiv$ constant and conclude that $\eta \equiv 0$ on the interval $(\alpha, \beta)$.

If $\eta^{\prime \prime}(x) \not \equiv 0$, set $U=\left\{x \in(\alpha, \beta): \eta^{\prime \prime}(x) \neq 0\right\}$. Then $U$ is a non-empty open set which implies that there exist $x_{0} \in U$ and some open set $O_{x_{0}}$ of $x_{0}$ contained in $U$. Then $\eta^{\prime \prime}(\xi) \neq$ 0 for all $\xi \in O_{x_{0}} \subset U$. Thus

$$
\frac{d}{d x}\left(\eta^{2}\right)=0 \quad \text { on } 0_{x_{0}}
$$

Hence, $\eta(\xi) \equiv$ constant on $O_{x_{0}}$. But this creates a contradiction because $\eta^{\prime \prime}(\xi) \equiv 0$ on $O_{x_{0}}$. Therefore,

$$
J[f+\eta]-J[f]=\delta J[f, \eta]
$$

only if $\eta(x) \equiv 0$, as desired. This completes the proof of the proposition. 
Corollary 3.2. Let $J[f]$ be as in (3.4). Then $f_{1} \in H_{0}^{2}([-1,1])$ is the unique minimizer for $J[f]$ if and only if $f_{1}$ solves the following ODE:

$$
\left\|g_{0}\right\|_{L^{2}}^{2} \frac{d^{4} f}{d x^{4}}-2\left\|g_{0}^{\prime}\right\|_{L^{2}}^{2} \frac{d^{2} f}{d x^{2}}+\left\|g_{0}^{\prime \prime}\right\|_{L^{2}}^{2} f=\gamma \int_{-1}^{1} g_{0} d y .
$$

Proof. Suppose $f_{1}$ is the unique minimizer. Then $f_{1}$ is a local extremum of $J[f]$. This implies that $\delta J[f, \eta]=0$ for all $\eta \in H_{0}^{2}([-1,1])$. Using the notations in (3.4), we have

$$
\begin{aligned}
0 & =\delta J[f, \eta] \\
& =\int_{-1}^{1}\left[\frac{\partial K}{\partial z} \eta+\frac{\partial K}{\partial V} \eta^{\prime}+\frac{\partial K}{\partial W} \eta^{\prime \prime}\right] d x \\
& =\int_{-1}^{1}\left[\frac{\partial K}{\partial z}-\frac{d}{d x}\left(\frac{\partial K}{\partial V}\right)+\frac{d^{2}}{d x^{2}}\left(\frac{\partial K}{\partial W}\right)\right] \eta(x) d x
\end{aligned}
$$

for all $\eta \in H_{0}^{2}([-1,1])$. This implies that

$$
\frac{\partial K}{\partial z}-\frac{d}{d x}\left(\frac{\partial K}{\partial V}\right)+\frac{d^{2}}{d x^{2}}\left(\frac{\partial K}{\partial W}\right)=0
$$

which is the Euler-Lagrange equation (3.18). This also follows from Lemma 2.1 directly.

Conversely, assume $f_{1}$ solves (3.18). Then the above argument shows that $\delta J[f, \eta]=0$ for all $\eta \in H_{0}^{2}([-1,1])$. Since $K$ satisfies condition (3.9) in Proposition 3.1, we conclude that

$$
J\left[f_{1}+\eta\right]-J\left[f_{1}\right] \geq \delta J\left[f_{1}, \eta\right], \quad \forall \eta \in C_{c}^{\infty}([-1,1]) .
$$

This tells us that $J\left[f_{1}+\eta\right] \geq J\left[f_{1}\right]$ for all $\eta \in C_{c}^{\infty}([-1,1])$ and $J\left[f_{1}+\eta\right]>J\left[f_{1}\right]$ if $\eta \not \equiv 0$. Observe that $J: H_{0}^{1}([-1,1]) \rightarrow \mathbb{R}$ as given in (3.4) is a continuous linear functional in the $H_{0}^{2}$-norm. This fact, combined with the density of $C_{c}^{\infty}([-1,1])$ in $H_{0}^{2}([-1,1])$ (in the $H_{0}^{2}$-norm), implies that

$$
J\left[f_{1}+\eta\right] \geq J\left[f_{1}\right], \quad \forall \eta \in C_{c}^{\infty}([-1,1]) .
$$

This means that for all $\varphi \in H_{0}^{2}([-1,1])$, we have $J[\varphi] \geq J\left[f_{1}\right]$ and if $\varphi \not \equiv f_{1}$ (almost everywhere), then $\varphi-f_{1} \neq \equiv$ and hence, $J[\varphi]>J\left[f_{1}\right]$. Thus $f_{1}$ is the unique minimum for $J$.

Reversing the roles of $f$ and $g$, that is, fixing $f_{0}$ and finding $g_{1} \in H_{0}^{2}$ to minimize $I\left[f_{0} g\right]$ over $g \in H_{0}^{2}([-1,1])$, we obtain the same conclusion by using the same arguments.

Corollary 3.3. Fix $f_{0} \in H_{0}^{2}([-1,1])$. Then $g_{1} \in H_{0}^{2}([-1,1])$ is the unique minimizer for

$$
\begin{aligned}
J[g]=I\left[f_{0} g\right]= & \left\|f_{0}\right\|_{L^{2}}^{2} \int_{-1}^{1}\left(g^{\prime \prime}\right)^{2} d y+2\left\|f_{0}^{\prime}\right\|_{L^{2}}^{2} \int_{-1}^{1}\left(g^{\prime}\right)^{2} d y \\
& +\left\|f_{0}^{\prime \prime}\right\|_{L^{2}}^{2} \int_{-1}^{1} g^{2} d y-2 \gamma\left\|f_{0}\right\|_{L^{1}} \int_{-1}^{1} g d y
\end{aligned}
$$


if and only if $g_{1}$ solves the Euler-Lagrange equation

$$
\left\|f_{0}\right\|_{L^{2}}^{2} \frac{d^{4} g}{d y^{4}}-2\left\|f_{0}^{\prime}\right\|_{L^{2}}^{2} \frac{d^{2} g}{d y^{2}}+\left\|f_{0}^{\prime \prime}\right\|_{L^{2}}^{2} g=2 \gamma \int_{-1}^{1} f_{0}(x) d x .
$$

Now we search for the solution $f_{1} \in H_{0}^{2}([-1,1])$ in $(3.18)$, that is,

$$
\left\|g_{0}\right\|_{L^{2}}^{2} \frac{d^{4} f}{d x^{4}}-2\left\|g_{0}^{\prime}\right\|_{L^{2}}^{2} \frac{d^{2} f}{d x^{2}}+\left\|g_{0}^{\prime \prime}\right\|_{L^{2}}^{2} f=2 \gamma \int_{-1}^{1} g_{0}(y) d y .
$$

Rewrite the above ODE in the following form:

$$
\left\|g_{0}\right\|_{L^{2}}^{2}\left[\left(D-\frac{\left\|g^{\prime}\right\|_{L^{2}}^{2}}{\left\|g_{0}\right\|_{L^{2}}^{2}}\right)^{2}+\frac{\left\|g^{\prime \prime}\right\|_{L^{2}}^{2}}{\left\|g_{0}\right\|_{L^{2}}^{2}}-\frac{\left\|g^{\prime}\right\|_{L^{2}}^{4}}{\left\|g_{0}\right\|_{L^{2}}^{4}}\right] f=2 \gamma \int_{-1}^{1} g_{0}(y) d y,
$$

where $D=d^{2} / d x^{2}$.

Remark 3.4. In general when $g \in H^{2}$, that is, $g$ needs not satisfy the zero boundary conditions for function in $H_{0}^{2}$, then the quantity

$$
\left(\frac{\left\|g_{0}^{\prime \prime}\right\|_{L^{2}}^{2}}{\left\|g_{0}\right\|_{L^{2}}^{2}}-\frac{\left\|g_{0}^{\prime}\right\|_{L^{2}}^{4}}{\left\|g_{0}\right\|_{L^{2}}^{4}}\right)
$$

can take on any values. However, if $g \in H_{0}^{2}$ and $g_{0} \neq \equiv 0$, as proved below, this quantity is always positive.

Lemma 3.5. Let $\Omega$ be a Lipschitz domain in $\mathbb{R}^{n}, n \geq 1$. Let $g \in H_{0}^{2}(\Omega)$ be arbitrary. Then

$$
\|\nabla g\|_{L^{2}}^{2} \leq\|g\|_{L^{2}} \cdot\|\Delta g\|_{L^{2}}
$$

and equality holds if and only if $g \equiv 0$.

Proof. Integration by parts yields

$$
\|\nabla g\|_{L^{2}}^{2}=\int_{\Omega} \nabla g \cdot \nabla g d \mathbf{x}=-\int_{\Omega} g \Delta g d \mathbf{x}+\int_{\partial \Omega} g \frac{\partial g}{\partial \vec{n}} d \sigma=-\int_{\Omega} g \Delta g d \mathbf{x} .
$$

By the Cauchy-Schwartz inequality, we have

$$
\|\nabla g\|_{L^{2}}^{2} \leq\|g\|_{L^{2}} \cdot\|\Delta g\|_{L^{2}}
$$

and the equality holds if and only if (see Lieb-Loss [9])

(i) $|g(\mathbf{x})|=\lambda|\Delta g(\mathbf{x})|$ almost everywhere for some $\lambda>0$,

(ii) $g(\mathbf{x}) \Delta g(\mathbf{x})=e^{i \theta}|g(\mathbf{x})| \cdot|\Delta g(\mathbf{x})|$.

Since $g$ is real-valued, (i) and (ii) imply

$$
g(\mathbf{x}) \Delta g(\mathbf{x})=\lambda(\Delta g(\mathbf{x}))^{2} .
$$


12 Poincaré inequality and Kantorovich method

So, $g$ must satisfy the following PDE:

$$
\Delta g-\frac{1}{\lambda} g=0
$$

where $g \in H_{0}^{2}(\Omega)$. But the only solution to this PDE is $g \equiv 0$ (see, Evans [3, pages 300$302])$. This completes the proof of the lemma.

Remark 3.6. If $n=1$, one can solve $g^{\prime \prime}-\lambda^{-1} g=0$ directly without having to appeal to the theory of elliptic PDEs.

Proposition 3.7. The solutions of (3.18) and (3.24) have the same form.

Proof. Using either Lemma 3.5 in case $n=1$ to the above remark, we see that

$$
\frac{\left\|g^{\prime \prime}\right\|_{L^{2}}^{2}}{\left\|g_{0}\right\|_{L^{2}}^{2}}-\frac{\left\|g^{\prime}\right\|_{L^{2}}^{4}}{\left\|g_{0}\right\|_{L^{2}}^{4}}>0 \quad \text { if } g_{0} \neq \equiv
$$

Hence the characteristic polynomial associated to (3.26) has two pairs of complex conjugate roots as long as $g_{0} \not \equiv 0$. Apply the same arguments to the ODE in (3.24) and the proposition is proved.

Remark 3.8. The statement in Proposition 3.7 was claimed in [8] without verification. Indeed the authors stated therein that the solutions of (3.18) and (3.24) are of the same form because of the positivity of the coefficients on the left-hand side of (3.18) and (3.24). As observed in Remark 3.4 and proved in Proposition 3.7, the positivity requirement is not sufficient. The fact that $f_{0}, g_{0} \in H_{0}^{2}$ must be used to conclude this assumption.

\section{Explicit solution for (3.26)}

We now find the explicit solution for (3.26), and hence for (3.18). Let

$$
\begin{array}{cc}
r=\frac{\left\|g^{\prime}\right\|_{L^{2}}}{\left\|g_{0}\right\|_{L^{2}}}, & t=\frac{\left\|g^{\prime \prime}\right\|_{L^{2}}}{\left\|g_{0}\right\|_{L^{2}}}, \\
\rho=\sqrt{\frac{t+r^{2}}{2}}, & \kappa=\sqrt{\frac{t-r^{2}}{2}} .
\end{array}
$$

Then from Proposition 3.7 and its proof, the 4 roots of the characteristic polynomial associated to ODE (3.26) are

$$
\rho+i \kappa, \quad \rho-i \kappa, \quad-\rho-i \kappa, \quad-\rho+i \kappa
$$

Thus the homogeneous solution of (3.26) is

$$
\begin{aligned}
f_{h}(x)= & c_{1} \cosh (\rho x) \cos (\kappa x)+c_{2} \sinh (\rho x) \cos (\kappa x) \\
& +c_{3} \cosh (\rho x) \sin (\kappa x)+c_{4} \sinh (\rho x) \sin (\kappa x) .
\end{aligned}
$$


It follows that a particular solution of $(3.26)$ is

$$
f_{p}(x)=\frac{2 \gamma \int_{-1}^{1} g_{0}(y) d y}{\left\|g_{0}^{\prime \prime}\right\|_{L^{2}}^{2}} .
$$

Thus the solution of (3.18) is

$$
\begin{aligned}
f(x)= & c_{1} \cosh (\rho x) \cos (\kappa x)+c_{2} \sinh (\rho x) \cos (\kappa x) \\
& +c_{3} \cosh (\rho x) \sin (\kappa x)+c_{4} \sinh (\rho x) \sin (\kappa x)+c_{p},
\end{aligned}
$$

where $c_{p}=2 \gamma \int_{-1}^{1} g_{0}(y) d y /\left\|g_{0}^{\prime \prime}\right\|_{L^{2}}^{2}$ is a known constant. This implies that

$$
\begin{aligned}
f^{\prime}(x)= & \rho c_{1} \sinh (\rho x) \cos (\kappa x)-\kappa c_{1} \cosh (\rho x) \sin (\kappa x) \\
& +\rho c_{2} \cosh (\rho x) \cos (\kappa x)-\kappa c_{2} \sinh (\rho x) \sin (\kappa x) \\
& +\rho c_{3} \sinh (\rho x) \sin (\kappa x)+\kappa c_{3} \cosh (\rho x) \cos (\kappa x) \\
& +\rho c_{4} \cosh (\rho x) \sin (\kappa x)+\kappa c_{4} \sinh (\rho x) \cos (\kappa x) .
\end{aligned}
$$

Apply the boundary conditions $f(1)=f(-1)=f^{\prime}(1)=f^{\prime}(-1)=0$, we get

$$
\begin{aligned}
& c_{1} \cosh (\rho) \cos (\kappa)+c_{2} \sinh (\rho) \cos (\kappa)+c_{3} \cosh (\rho) \sin (\kappa)+c_{4} \sinh (\rho) \sin (\kappa)=-c_{p}, \\
& c_{1} \cosh (\rho) \cos (\kappa)-c_{2} \sinh (\rho) \cos (\kappa)-c_{3} \cosh (\rho) \sin (\kappa)+c_{4} \sinh (\rho) \sin (\kappa)=-c_{p}, \\
& c_{1}[\rho \sinh (\rho) \cos (\kappa)-\kappa \cosh (\rho) \sin (\kappa)]+c_{2}[\rho \cosh (\rho) \cos (\kappa)-\kappa \sinh (\rho) \sin (\kappa)] \\
& \quad+c_{3}[\rho \sinh (\rho) \sin (\kappa)+\kappa \cosh (\rho) \cos (\kappa)]+c_{4}[\rho \cosh (\rho) \sin (\kappa)+\kappa \sinh (\rho) \cos (\kappa)]=0, \\
& c_{1}[-\rho \sinh (\rho) \cos (\kappa)+\kappa \cosh (\rho) \sin (\kappa)]+c_{2}[\rho \cosh (\rho) \cos (\kappa)-\kappa \sinh (\rho) \sin (\kappa)] \\
& \quad+c_{3}[\rho \sinh (\rho) \sin (\kappa)+\kappa \cosh (\rho) \cos (\kappa)]-c_{4}[\rho \cosh (\rho) \sin (\kappa)+\kappa \sinh (\rho) \cos (\kappa)]=0 .
\end{aligned}
$$

Hence,

$$
\begin{gathered}
c_{1} \cosh (\rho) \cos (\kappa)+c_{4} \sinh (\rho) \sin (\kappa)=-c_{p}, \\
c_{2} \sinh (\rho) \cos (\kappa)+c_{3} \cosh (\rho) \sin (\kappa)=0, \\
c_{2}[\rho \cosh (\rho) \cos (\kappa)-\kappa \sinh (\rho) \sin (\kappa)] \\
+c_{3}[\rho \sinh (\rho) \sin (\kappa)+\kappa \cosh (\rho) \cos (\kappa)]=0, \\
c_{1}[\rho \sinh (\rho) \cos (\kappa)-\kappa \cosh (\rho) \sin (\kappa)] \\
+c_{4}[\rho \cosh (\rho) \sin (\kappa)+\kappa \sinh (\rho) \cos (\kappa)]=0 .
\end{gathered}
$$

We know, beforehand, that there must be a unique solution. Thus (4.9) and (4.23) force 
14 Poincaré inequality and Kantorovich method

$c_{2}=c_{3}=0$. We are left to solve for $c_{1}$ and $c_{4}$ from (4.8) and (4.11). But (4.11) tells us that

$$
c_{1}=-c_{4} \frac{\rho \cosh (\rho) \sin (\kappa)+\kappa \sinh (\rho) \cos (\kappa)}{\rho \sinh (\rho) \cos (\kappa)-\kappa \cosh (\rho) \sin (\kappa)} .
$$

Substituting (4.12) into (4.8), we have

$$
c_{4}=c_{p} \frac{\rho \sinh (\rho) \cos (\kappa)-\kappa \cosh (\rho) \sin (\kappa)}{\rho \sin (\kappa) \cos (\kappa)+\kappa \sinh (\rho) \cosh (\rho)} .
$$

Plugging (4.13) into (4.12), we have

$$
c_{1}=-c_{p} \frac{\rho \cosh (\rho) \sin (\kappa)+\kappa \sinh (\rho) \cos (\kappa)}{\rho \sin (\kappa) \cos (\kappa)+\kappa \sinh (\rho) \cosh (\rho)} .
$$

Therefore, the solution $f_{1}(x)$ can be written in the form

$$
f_{1}(x)=c_{p}\left[\frac{K_{1}}{K_{0}} \cosh (\rho x) \cos (\kappa x)+\frac{K_{2}}{K_{0}} \sinh (\rho x) \sin (\kappa x)+1\right]
$$

where

$$
\begin{gathered}
c_{p}=\frac{2 \gamma \int_{-1}^{1} g_{0}(y) d y}{\left\|g_{0}^{\prime \prime}\right\|_{L^{2}}^{2}}, \\
\rho=\sqrt{\frac{t+r^{2}}{2}}=\sqrt{\frac{\left\|g_{0}^{\prime \prime}\right\| L_{L^{2}} /\left\|g_{0}\right\|_{L^{2}}+\left\|g_{0}^{\prime}\right\|_{L^{2}}^{2} /\left\|g_{0}\right\|_{L^{2}}^{2}}{2}}, \\
\kappa=\sqrt{\frac{t-r^{2}}{2}}=\sqrt{\frac{\left\|g_{0}^{\prime \prime}\right\| L_{L^{2}} /\left\|g_{0}\right\|_{L^{2}}-\left\|g_{0}^{\prime}\right\|_{L^{2}}^{2} /\left\|g_{0}\right\|_{L^{2}}^{2}}{2}}, \\
K_{0}=\rho \sin (\kappa) \cos (\kappa)+\kappa \sinh (\rho) \cosh (\rho), \\
K_{1}=-\rho \cosh (\rho) \sin (\kappa)-\kappa \sinh (\rho) \cos (\kappa), \\
K_{2}=\rho \sinh (\rho) \cos (\kappa)-\kappa \cosh (\rho) \sin (\kappa) .
\end{gathered}
$$

The next step in the extended Kantorovich method is to fix $f_{1}(x)$ just found above and solve for $g_{1}(y) \in H_{0}^{2}([-1,1])$ from (3.24). Lemma 2.2 and the computation above show that

$$
g_{1}(y)=\widetilde{c}_{p}\left[\frac{\widetilde{K}_{1}}{\widetilde{K}_{0}} \cosh (\tilde{\rho} y) \cos (\tilde{\kappa} y)+\frac{\widetilde{K}_{2}}{\widetilde{K}_{0}} \sinh (\tilde{\rho} y) \sin (\tilde{\kappa} y)+1\right],
$$


where

$$
\begin{gathered}
\tilde{c}_{p}=\frac{2 \gamma \int_{-1}^{1} f_{1}(x) d x}{\left\|f_{1}^{\prime \prime}\right\|_{L^{2}}^{2}}, \\
\tilde{\rho}=\sqrt{\frac{\left\|f_{1}^{\prime \prime}\right\|_{L^{2}} /\left\|f_{1}\right\|_{L^{2}}+\left\|f_{1}^{\prime}\right\|_{L^{2}}^{2} /\left\|f_{1}\right\|_{L^{2}}^{2}}{2}}, \\
\tilde{\kappa}=\sqrt{\frac{\left\|f_{1}^{\prime \prime}\right\|_{L^{2}} /\left\|f_{1}\right\|_{L^{2}}-\left\|f_{1}^{\prime}\right\|_{L^{2}}^{2} /\left\|f_{1}\right\|_{L^{2}}^{2}}{2}} \\
\tilde{K}_{0}=\tilde{\rho} \sin (\tilde{\kappa}) \cos (\tilde{\kappa})+\tilde{\kappa} \sinh (\tilde{\rho}) \cosh (\tilde{\rho}), \\
\tilde{K}_{1}=-\tilde{\rho} \cosh (\tilde{\rho}) \sin (\tilde{\kappa})-\tilde{\kappa} \sinh (\tilde{\rho}) \cos (\tilde{\kappa}), \\
\tilde{K}_{2}=\tilde{\rho} \sinh (\tilde{\rho}) \cos (\tilde{\kappa})-\tilde{\kappa} \cosh (\tilde{\rho}) \sin (\tilde{\kappa}) .
\end{gathered}
$$

Now we start the next iteration by fixing $g_{1}(y)$ and solving for $f_{2}(x)$ in (3.18), and so forth. In particular, we will write

$$
f_{n}(x)=c_{n}\left[\frac{K_{1 n}}{K_{0 n}} \cosh \left(\rho_{n} x\right) \cos \left(\kappa_{n} x\right)+\frac{K_{2 n}}{K_{0 n}} \sinh \left(\rho_{n} x\right) \sin \left(\kappa_{n} x\right)+1\right],
$$

where

$$
\begin{gathered}
c_{n}=\frac{2 \gamma \int_{-1}^{1} g_{n-1}(y) d y}{\left\|g_{n-1}^{\prime \prime}\right\|_{L^{2}}^{2}}, \\
\rho_{n}=\sqrt{\frac{\left\|g_{n-1}^{\prime \prime}\right\|_{L^{2}} /\left\|g_{n-1}\right\|_{L^{2}}+\left\|g_{n-1}^{\prime}\right\|_{L^{2}}^{2} /\left\|g_{n-1}\right\|_{L^{2}}^{2}}{2}}, \\
\kappa_{n}=\sqrt{\frac{\left\|g_{n-1}^{\prime \prime}\right\|_{L^{2}} /\left\|g_{n-1}\right\|_{L^{2}}-\left\|g_{n-1}^{\prime}\right\|_{L^{2}}^{2} /\left\|g_{n-1}\right\|_{L^{2}}^{2}}{2}}, \\
K_{0 n}=\rho_{n} \sin \left(\kappa_{n}\right) \cos \left(\kappa_{n}\right)+\kappa_{n} \sinh \left(\rho_{n}\right) \cosh \left(\rho_{n}\right), \\
K_{1 n}=-\rho_{n} \cosh \left(\rho_{n}\right) \sin \left(\kappa_{n}\right)-\kappa_{n} \sinh \left(\rho_{n}\right) \cos \left(\kappa_{n}\right), \\
K_{2 n}=\rho_{n} \sinh \left(\rho_{n}\right) \cos \left(\kappa_{n}\right)-\kappa_{n} \cosh \left(\rho_{n}\right) \sin \left(\kappa_{n}\right) .
\end{gathered}
$$

Similarly,

$$
g_{n}(y)=\widetilde{c}_{n}\left[\frac{\widetilde{K}_{1 n}}{\widetilde{K}_{0 n}} \cosh \left(\tilde{\rho}_{n} y\right) \cos \left(\widetilde{\kappa}_{n} y\right)+\frac{\widetilde{K}_{2 n}}{\widetilde{K}_{0 n}} \sinh \left(\tilde{\rho}_{n} y\right) \sin \left(\widetilde{\kappa}_{n} y\right)+1\right],
$$


16 Poincaré inequality and Kantorovich method

where

$$
\begin{gathered}
\tilde{c}_{n}=\frac{2 \gamma \int_{-1}^{1} f_{n}(x) d x}{\left\|f_{n}^{\prime \prime}\right\|_{L^{2}}^{2}}, \\
\tilde{\rho}=\sqrt{\frac{\left\|f_{n}^{\prime \prime}\right\|_{L^{2}} /\left\|f_{n}\right\|_{L^{2}}+\left\|f_{n}^{\prime}\right\|_{L^{2}}^{2} /\left\|f_{n}\right\|_{L^{2}}^{2}}{2}}, \\
\tilde{\kappa}_{n}=\sqrt{\frac{\left\|f_{n}^{\prime \prime}\right\|_{L^{2}} /\left\|f_{n}\right\|_{L^{2}}-\left\|f_{n}^{\prime}\right\|_{L^{2}}^{2} /\left\|f_{n}\right\|_{L^{2}}^{2}}{2}} \\
\tilde{K}_{0 n}=\tilde{\rho}_{n} \sin \left(\tilde{\kappa}_{n}\right) \cos \left(\tilde{\kappa}_{n}\right)+\tilde{\kappa}_{n} \sinh \left(\tilde{\rho}_{n}\right) \cosh \left(\tilde{\rho}_{n}\right), \\
\tilde{K}_{1 n}=-\tilde{\rho}_{n} \cosh \left(\tilde{\rho}_{n}\right) \sin \left(\tilde{\kappa}_{n}\right)-\tilde{\kappa}_{n} \sinh \left(\tilde{\rho}_{n}\right) \cos \left(\tilde{\kappa}_{n}\right), \\
\tilde{K}_{2 n}=\tilde{\rho}_{n} \sinh \left(\tilde{\rho}_{n}\right) \cos \left(\tilde{\kappa}_{n}\right)-\tilde{\kappa}_{n} \cosh \left(\tilde{\rho}_{n}\right) \sin \left(\tilde{\kappa}_{n}\right) .
\end{gathered}
$$

In summary, a solution $\phi_{n}(x, y)$ in Lemma 2.3 can be written into the following form:

$$
\begin{aligned}
\phi_{n}(x, y)=f_{n}(x) & g_{n}(y) \\
=c_{n} \widetilde{c}_{n}[ & \frac{K_{1 n} \widetilde{K}_{1 n}}{K_{0 n} \widetilde{K}_{0 n}} \cosh \left(\rho_{n} x\right) \cosh \left(\tilde{\rho}_{n} y\right) \cos \left(\kappa_{n} x\right) \cos \left(\widetilde{\kappa}_{n} y\right) \\
& +\frac{K_{1 n} \widetilde{K}_{2 n}}{K_{0 n} \widetilde{K}_{0 n}} \cosh \left(\rho_{n} x\right) \sinh \left(\tilde{\rho}_{n} y\right) \cos \left(\kappa_{n} x\right) \sin \left(\widetilde{\kappa}_{n} y\right) \\
& +\frac{K_{2 n} \widetilde{K}_{1 n}}{K_{0 n} \widetilde{K}_{0 n}} \sinh \left(\rho_{n} x\right) \cosh \left(\tilde{\rho}_{n} y\right) \sin \left(\kappa_{n} x\right) \cos \left(\widetilde{\kappa}_{n} y\right) \\
& +\frac{K_{2 n} \widetilde{K}_{2 n}}{K_{0 n} \widetilde{K}_{0 n}} \sinh \left(\rho_{n} x\right) \sinh \left(\tilde{\rho}_{n} y\right) \sin \left(\kappa_{n} x\right) \cos \left(\widetilde{\kappa}_{n} y\right) \\
& +\frac{K_{1 n}}{K_{0 n}} \cosh \left(\rho_{n} x\right) \cos \left(\kappa_{n} x\right)+\frac{K_{2 n}}{K_{0 n}} \sinh \left(\rho_{n} x\right) \sin \left(\kappa_{n} x\right) \\
& \left.+\frac{\widetilde{K}_{1 n}}{\widetilde{K}_{0 n}} \cosh \left(\tilde{\rho}_{n} y\right) \sin \left(\widetilde{\kappa}_{n} y\right)+\frac{\widetilde{K}_{2 n}}{\widetilde{K}_{0 n}} \sinh \left(\tilde{\rho}_{n} y\right) \sin \left(\widetilde{\kappa}_{n} y\right)+1\right] .
\end{aligned}
$$

\section{Convergence of the solutions}

In order to discuss the convergence of the extended Kantorovich method, let us start with the following auxiliary lemma.

Lemma 5.1. Let $\phi_{n}(x, y)=f_{n}(x) g_{n}(y)$ and $\psi_{n}(x, y)=f_{n+1}(x) g_{n}(y)$. Then these two sequences are bounded in $H_{0}^{2}(\Omega)$.

Proof. We will verify the boundedness of $\left\{\psi_{n}\right\}$ for the arguments which is identical for the sequence $\left\{\phi_{n}\right\}$. Fix an integer $n \in \mathbf{Z}_{+}$and assume that $g_{n}$ has been determined from the extended Kantorovich scheme when $n \geq 1$ or $g_{n}$ has been chosen a priori when $n=0$. 
Then $f_{n+1}$ is determined by minimizing

$$
\begin{aligned}
I\left[f g_{n}\right]= & J[f] \\
= & \left\|g_{n}\right\|_{L^{2}}^{2} \int\left(f^{\prime \prime}\right)^{2} d x+2\left\|g_{n}^{\prime}\right\|_{L^{2}}^{2} \int\left(f^{\prime}\right)^{2} d x \\
& +\left\|g_{n}^{\prime \prime}\right\|_{L^{2}}^{2} \int f^{2} d x-2 \gamma \int g_{n} d y \cdot \int f d x .
\end{aligned}
$$

By Corollary 3.2, if $f_{n+1}$ is as in (4.19), then $f_{n+1}$ is the unique minimum for $J[f]$ over $H_{0}^{2}(\Omega)$. Thus we must have

$$
I\left[f_{n+1} g_{n}\right]=I\left[f_{n+1}\right]<I[0]=0 .
$$

This implies that

$$
\int_{\Omega}\left|\Delta \psi_{n}\right|^{2}-\gamma \int_{\Omega} \psi_{n} d x d y<0
$$

Lemma 2.2 then yields

$$
\left\|\psi_{n}\right\|_{H_{0}^{2}(\Omega)}^{2}<C \gamma\left\|\psi_{n}\right\|_{L^{2}(\Omega)}^{2}<C \gamma\left\|\psi_{n}\right\|_{H_{0}^{2}(\Omega)} .
$$

Therefore, $\left\|\psi_{n}\right\|_{H_{0}^{2}(\Omega)}<C \gamma$ as desired.

Now we are in a position to prove the main theorem of this section.

Theorem 5.2. There exist subsequences $\left\{\phi_{n_{j}}\right\}_{j}$ and $\left\{\psi_{n_{j}}\right\}_{j}$ of $\left\{\phi_{n}\right\}$ and $\left\{\psi_{n}\right\}$ which converge in $L^{2}(\Omega)$ to some functions $\phi, \psi \in H_{0}^{2}(\Omega)$. Furthermore if

$$
\mathscr{L}=\left\{g \in H_{0}^{2}([-1,1]): \int_{-1}^{1} g(y) d y=0\right\}
$$

and if $g_{0} \notin \mathscr{L}$, then

$$
\lim _{j}\left\|\phi_{n_{j}}\right\|_{L^{2}}>0, \quad \quad \lim _{j}\left\|\psi_{n_{j}}\right\|_{L^{2}}>0, \quad \quad \lim _{j}\left\|\phi_{n_{j}}\right\|_{L^{1}}>0, \quad \quad \lim _{j}\left\|\psi_{n_{j}}\right\|_{L^{1}}>0 .
$$

Therefore, the above limits are zero if and only if $g_{0} \in \mathscr{L}$.

Proof. From Lemma 5.1, $\left\{\phi_{n}\right\}$ and $\left\{\psi_{n}\right\}$ are bounded in $H_{0}^{2}(\Omega)$. As a consequence of a weak compactness theorem, there are subsequences $\left\{\phi_{n_{j}}\right\}$ and $\left\{\psi_{n_{j}}\right\}$ and functions $\phi$ and $\psi$ in $H_{0}^{2}(\Omega)$ such that

$$
\phi_{n_{j}} \longrightarrow \phi, \quad \psi_{n_{j}} \longrightarrow \psi, \quad \text { weakly in } H_{0}^{2}(\Omega)
$$

By the Sobolev embedding theorem on the compact embedding of $H_{0}^{1}(\Omega)$ in $L^{2}(\Omega)$, we conclude that after passing to another subsequence if necessary,

$$
\phi_{n_{j}} \longrightarrow \phi, \quad \psi_{n_{j}} \longrightarrow \psi, \quad \text { in } L^{2}(\Omega) .
$$


18 Poincaré inequality and Kantorovich method

From (4.19), we see that $g_{0} \in \mathscr{L}$ if and only if $f_{1} \equiv 0$. Hence if $g_{0} \in \mathscr{L}$, the iteration process of the extended Kantorovich method stops and we have $\psi_{1}(x, y)=f_{1}(x) g_{0}(y) \equiv 0$. Now suppose $g_{0} \notin \mathscr{L}$, that is, $f_{1} \not \equiv 0$. As in the proof of Lemma 5.1, Corollary 3.2 implies that

$$
I\left[f_{1} g_{0}\right]<I[0]=0,
$$

since $f_{1}$ is the unique minimizer of $I\left[f g_{0}\right]$ and $f_{1} \not \equiv 0$. Applying Corollary 3.2 repeatedly, one has

$$
I\left[f_{m+1} g_{m}\right]<\cdots<I\left[f_{2} g_{1}\right]<I\left[f_{1} g_{1}\right]<I\left[f_{1} g_{0}\right]<0 .
$$

But by Lemma 2.3,

$$
I[\psi] \leq \liminf _{j} I\left[\psi_{n_{j}}\right]:=\liminf _{j} I\left[f_{n_{j}+1} g_{n_{j}}\right]
$$

In view of (5.10), we must have $J[\psi]<0$, which implies $\lim _{j}\left\|\psi_{n_{j}}\right\|_{L^{2}}=\|\psi\|_{L^{2}}>0$; otherwise, we would have $\|\psi\|_{L^{2}}=0$ which implies that $J[\psi]=0$. Similarly, $\lim _{j}\left\|\phi_{n_{j}}\right\|_{L^{2}}=$ $\|\phi\|_{L^{2}}>0$. Since $\psi_{n_{j}} \rightarrow \psi$ and $\phi_{n_{j}} \rightarrow \phi$ in $L^{2}$, we also have $\psi_{n_{j}} \rightarrow \psi$ and $\phi_{n_{j}} \rightarrow \phi$ in $L^{1}$. Thus

$$
\lim _{j}\left\|\psi_{n_{j}}\right\|_{L^{1}}=\|\psi\|_{L^{1}}>0, \quad \lim _{j}\left\|\psi_{n_{j}}\right\|_{L^{1}}=\|\psi\|_{L^{1}}>0
$$

This completes the proof of the proposition.

Corollary 5.3. Let $g_{0} \notin \mathscr{L}$ and set

$$
r_{n}=\frac{\left\|g_{n-1}^{\prime}\right\|_{L^{2}}}{\left\|g_{n-1}\right\|_{L^{2}}}, \quad \tilde{r}_{n}=\frac{\left\|f_{n}^{\prime}\right\|_{L^{2}}}{\left\|f_{n}\right\|_{L^{2}}}, \quad t_{n}=\frac{\left\|g_{n-1}^{\prime \prime}\right\|_{L^{2}}}{\left\|g_{n-1}\right\|_{L^{2}}}, \quad \tilde{t}_{n}=\frac{\left\|f_{n}^{\prime \prime}\right\|_{L^{2}}}{\left\|f_{n}\right\|_{L^{2}}} .
$$

Then there exist subsequences $\left\{f_{n_{j}}\right\}$ and $\left\{g_{n_{j}}\right\}$ such that the following limits exist and are positive:

$$
\lim _{j} r_{n_{j}}, \quad \lim _{j} \tilde{r}_{n_{j}}, \quad \lim _{j} t_{n_{j}}, \quad \lim _{j} \tilde{t}_{n_{j}}
$$

Proof. In the proof of Theorem 5.2, we showed that for each $n$,

$$
I\left[\phi_{n}\right]=\int_{\Omega}\left|\Delta \phi_{n}\right|^{2} d x d y-\gamma \phi_{n}<0
$$

as long as $g_{0} \notin \mathscr{L}$. Consequently,

$$
\left\|f_{n}^{\prime \prime}\right\|_{L^{2}}^{2}\left\|g_{n}\right\|_{L^{2}}^{2}+\left\|g_{n}^{\prime \prime}\right\|_{L^{2}}^{2}\left\|f_{n}\right\|_{L^{2}}^{2} \leq \gamma\left\|f_{n}\right\|_{L^{2}}^{2}\left\|g_{n}\right\|_{L^{2}}^{2} .
$$

This implies that

$$
\left\|f_{n}^{\prime \prime}\right\|_{L^{2}}^{2}\left\|g_{n}\right\|_{L^{2}}^{2} \leq \gamma\left\|f_{n} g_{n}\right\|_{L^{2}} \Longrightarrow \frac{\left\|f_{n}^{\prime \prime}\right\|_{L^{2}}^{2}}{\left\|f_{n}\right\|_{L^{2}}^{2}} \leq \frac{\gamma}{\left\|\phi_{n}\right\|_{L^{2}}}
$$


Combining with the Poincaré inequality, it follows that

$$
0<C^{\prime} \leq C \frac{\left\|f_{n}^{\prime \prime}\right\|_{L^{2}}^{2}}{\left\|f_{n}\right\|_{L^{2}}^{2}} \leq \frac{\left\|f_{n}^{\prime \prime}\right\|_{L^{2}}^{2}}{\left\|f_{n}\right\|_{L^{2}}^{2}} \leq \frac{\gamma}{\left\|\phi_{n}\right\|_{L^{2}}}
$$

for some universal constants $C$ and $C^{\prime}$. With Theorem 5.2, the above string of inequalities yields

$$
\begin{array}{ll}
\widetilde{C}_{1} \leq \limsup _{j} \tilde{r}_{n_{j}} \leq \widetilde{C}_{2}, & \widetilde{C}_{1} \leq \limsup _{j} \tilde{t}_{n_{j}} \leq \widetilde{C}_{2}, \\
\widetilde{C}_{1} \leq \liminf _{j} \tilde{r}_{n_{j}} \leq \widetilde{C}_{2}, & \widetilde{C}_{1} \leq \liminf _{j} \tilde{t}_{n_{j}} \leq \widetilde{C}_{2},
\end{array}
$$

for some positive constants $\widetilde{C}_{1}$ and $\widetilde{C}_{2}$. Similar inequalities hold for $r_{n_{j}}$ and $t_{n_{j}}$ with some positive constants $C_{1}$ and $C_{2}$. Thus after further extracting subsequences of $\left\{f_{n_{j}}\right\}$ and $\left\{g_{n_{j}}\right\}$, we may conclude that the following limits exist and are non-zero:

$$
\lim _{j} \frac{\left\|f_{n}^{\prime \prime}\right\|_{L^{2}}}{\left\|f_{n}\right\|_{L^{2}}}, \quad \lim _{j} \frac{\left\|f_{n}^{\prime}\right\|_{L^{2}}}{\left\|f_{n}\right\|_{L^{2}}}, \quad \lim _{j} \frac{\left\|g_{n}^{\prime \prime}\right\|_{L^{2}}}{\left\|g_{n}\right\|_{L^{2}}}, \quad \lim _{j} \frac{\left\|g_{n}^{\prime}\right\|_{L^{2}}}{\left\|g_{n}\right\|_{L^{2}}} .
$$

This completes the proof of the corollary.

Corollary 5.4. If $g_{0} \notin \mathscr{Z}$, then there exists a subsequence $\left\{f_{n_{j}} g_{n_{j}}\right\}_{j}$ that converges pointwisely to a function of the form

$$
\Theta(x, y)=\sum_{k=1}^{N} F_{k}(x) G_{k}(y) \in H_{0}^{2}(\Omega) .
$$

Furthermore, the derivatives of all orders of $\left\{f_{n_{j}} g_{n_{j}}\right\}_{j}$ also converge pointwisely to that of $F(x) G(y)$.

Proof. Let us observe the expression of $\phi_{n}(x, y)=f_{n}(x) g_{n}(y)$ in (4.23). Applying Corollary 5.3 to the constants on the right-hand side of (4.23), we can find convergent subsequences:

$$
\left\{K_{0 n_{j}}\right\},\left\{K_{1 n_{j}}\right\},\left\{K_{2 n_{j}}\right\},\left\{\widetilde{K}_{0 n_{j}}\right\},\left\{\widetilde{K}_{1 n_{j}}\right\},\left\{\widetilde{K}_{2 n_{j}}\right\},
$$

and $\left\{\rho_{n_{j}}\right\},\left\{\kappa_{n_{j}}\right\},\left\{\tilde{\rho}_{n_{j}}\right\},\left\{\tilde{\kappa}_{n_{j}}\right\}$. In addition, the constants $c_{n} \widetilde{c}_{n}$ can be rewritten as

$$
\begin{aligned}
c_{n} \widetilde{c}_{n} & =\frac{\gamma^{2} \int_{-1}^{1} g_{n-1}(y) d x \int_{-1}^{1} f_{n}(x) d x}{\left\|g_{n-1}^{\prime \prime}\right\|_{L^{2}}^{2}\left\|f_{n}^{\prime \prime}\right\|_{L^{2}}^{2}} \\
& =\frac{\gamma^{2} \int_{\Omega} f_{n}(x) g_{n-1}(y) d x d y}{\left\|f_{n} g_{n-1}\right\|_{L^{2}}^{2}} \cdot \frac{\left\|g_{n-1}\right\|_{L^{2}}^{2}}{\left\|g_{n-1}^{\prime \prime}\right\|_{L^{2}}^{2}} \cdot \frac{\left\|f_{n}\right\|_{L^{2}}^{2}}{\left\|f_{n}^{\prime \prime}\right\|_{L^{2}}^{2}} ;
\end{aligned}
$$

hence Theorem 5.2 and Corollary 5.3 guarantee the convergence of the subsequence $\left\{c_{n-1} \tilde{c}_{n-j}\right\}$. Altogether, after replacing all sequences on the right-hand side of (4.23) with 
either convergent subsequences, we get

$$
\begin{aligned}
\Theta(x, y)=\lim _{j} f_{n_{j}} g_{n_{j}} \\
=C\left\{\frac{K_{1 \infty} \widetilde{K}_{1 \infty}}{K_{0 \infty} \widetilde{K}_{0 \infty}} \cosh \left(\rho_{\infty} x\right) \cosh \left(\tilde{\rho}_{\infty} y\right) \cos \left(\kappa_{\infty} x\right) \cos \left(\tilde{\kappa}_{\infty} y\right)\right. \\
++\frac{K_{1 \infty} \widetilde{K}_{2 \infty}}{K_{0 \infty} \widetilde{K}_{0 \infty}} \cosh \left(\rho_{\infty} x\right) \sinh \left(\tilde{\rho}_{\infty} y\right) \cos \left(\kappa_{\infty} x\right) \sin \left(\widetilde{\kappa}_{\infty} y\right) \\
+\frac{K_{2 \infty} \widetilde{K}_{1 \infty}}{K_{0 \infty} \widetilde{K}_{0 \infty}} \sinh \left(\rho_{\infty} x\right) \cosh \left(\tilde{\rho}_{\infty} y\right) \sin \left(\kappa_{\infty} x\right) \cos \left(\widetilde{\kappa}_{\infty} y\right) \\
+\frac{K_{2 \infty} \widetilde{K}_{2 \infty}}{K_{0 \infty} \widetilde{K}_{0 \infty}} \sinh \left(\rho_{\infty} x\right) \sinh \left(\tilde{\rho}_{\infty} y\right) \sin \left(\kappa_{\infty} x\right) \cos \left(\widetilde{\kappa}_{\infty} y\right) \\
+\frac{K_{1 \infty}}{K_{0 \infty}} \cosh \left(\rho_{\infty} x\right) \cos \left(\kappa_{\infty} x\right)+\frac{K_{2 \infty}}{K_{0 \infty}} \sinh \left(\rho_{\infty} x\right) \sin \left(\kappa_{\infty} x\right) \\
\left.+\frac{\widetilde{K}_{1 \infty}}{\widetilde{K}_{0 \infty}} \cosh \left(\tilde{\rho}_{\infty} y\right) \sin \left(\widetilde{\kappa}_{\infty} y\right)+\frac{\widetilde{K}_{2 \infty}}{\widetilde{K}_{0 \infty}} \sinh \left(\tilde{\rho}_{\infty} y\right) \sin \left(\widetilde{\kappa}_{\infty} y\right)+1\right\} .
\end{aligned}
$$

Now if we differentiate $f_{n} g_{n}$ a finite number of times, then from (4.23) we have each summand scaled by integral powers of $\rho_{n}, \tilde{\rho}_{n}, \kappa_{n}$ and $\tilde{\kappa}_{n}$. But we just argued above that these sequences have convergent subsequences. Hence when $x, y$ are fixed, we conclude that all derivatives of $f_{n_{j}} g_{n_{j}}$ at $(x, y)$ will converge to that of $\Theta(x, y)$ as $k \rightarrow \infty$. The proof of the corollary is therefore complete.

Remark 5.5. Corollary 5.4 implies that

$$
I\left[f_{n_{j}} g_{n_{j}}\right] \longrightarrow I[\Theta(x, y)]
$$

by directly using the definition of $I[f g]$. Without Corollary 5.4, we can only assert that

$$
I[\Theta(x, y)] \leq \liminf _{j} I\left[f_{n_{j}} g_{n_{j}}\right] .
$$

\section{Acknowledgments}

The authors are grateful to the referee for helpful comments and a careful reading of the manuscript. The first author was partially supported by a William Fulbright Research Grant and a Competitive Research Grant at Georgetown University. The fourth author was partially supported by US Army Research Office Grant under the FY96 MURI in Active Control of Rotorcraft Vibration and Acoustics.

\section{References}

[1] D.-C. Chang, G. Wang, and N. M. Wereley, A generalized Kantorovich method and its application to free in-plane plate vibration problem, Applicable Analysis 80 (2001), no. 3-4, 493-523.

[2] Analysis and applications of extended Kantorovich-Krylov method, Applicable Analysis 82 (2003), no. 7, 713-740.

[3] L. C. Evans, Partial Differential Equations, Graduate Studies in Mathematics, vol. 19, American Mathematical Society, Rhode Island, 1998. 
[4] N. H. Farag and J. Pan, Model characteristics of in-plane vibration of rectangular plates, Journal of Acoustics Society of America 105 (1999), no. 6, 3295-3310.

[5] F. John, Partial Differential Equations, 3rd ed., Applied Mathematical Sciences, vol. 1, Springer, New York, 1978.

[6] L. V. Kantorovich and V. I. Krylov, Approximate Method of Higher Analysis, Noordhoff, Groningen, 1964.

[7] A. D. Kerr, An extension of the Kantorovich method, Quarterly of Applied Mathematics 26 (1968), no. 2, 219-229.

[8] A. D. Kerr and H. Alexander, An application of the extended Kantorovich method to the stress analysis of a clamped rectangular plate, Acta Mechanica 6 (1968), 180-196.

[9] E. H. Lieb and M. Loss, Analysis, Graduate Studies in Mathematics, vol. 14, American Mathematical Society, Rhode Island, 1997.

[10] E. M. Stein, Singular Integrals and Differentiability Properties of Functions, Princeton Mathematical Series, no. 30, Princeton University Press, New Jersey, 1970.

[11] G. Wang, N. M. Wereley, and D.-C. Chang, Analysis of sandwich plates with viscoelastic damping using two-dimensional plate modes, AIAA Journal 41 (2003), no. 5, 924-932.

[12] - Analysis of bending vibration of rectangular plates using two-dimensional plate modes, AIAA Journal of Aircraft 42 (2005), no. 2, 542-550.

Der-Chen Chang: Department of Mathematics, Georgetown University, Washington,

DC 20057-0001, USA

E-mail address: chang@math.georgetown.edu

Tristan Nguyen: Department of Defense, Fort Meade, MD 20755, USA

E-mail address: tristan@afterlife.ncsc.mil

Gang Wang: Smart Structures Laboratory, Alfred Gessow Rotorcraft Center,

Department of Aerospace Engineering, University of Maryland,

College Park, MD 20742, USA

E-mail address: gwang@eng.umd.edu

Norman M. Wereley: Smart Structures Laboratory, Alfred Gessow Rotorcraft Center, Department of Aerospace Engineering, University of Maryland,

College Park, MD 20742, USA

E-mail address: wereley@eng.umd.edu 